\title{
MEASUREMENTS OF THE VELOCITY FIELDS BY PIV METHOD ROUND ABOUT TITLING GATE
}

\begin{abstract}
Ivana MISTROVÁ, Pavel ZUBÍK•
Abstract: The article deals with problems of using of measurement method Particle Image Velocimetry (PIV) to measure velocity fields in the flowing water in front, above and behind drowned titling weir gate. The aim was to obtain information about the distribution of speed in the area of interest for the verification or calibration of the numerical model. Experiments were carried out in inclinable channel connected to the hydraulic circuit with a pump and storage tank at the Water Management Research Laboratory (LVV) of Institute of Water Structures at the Faculty of Civil Engineering in Brno University of Technology. Hydraulic inclinable channel has cross-section with dimensions of $0.4 \times 0.4 \mathrm{~m}$ and length of $12.5 \mathrm{~m}$. The measured area has cross-section approximately $0.2 \mathrm{~m}$ wide and $0.4 \mathrm{~m}$ high and its length is $1 \mathrm{~m}$. The results of physical modelling allowed a comparison of experimental data with numerical simulation results of this type of flow in the commercial software ANSYS CFX-12.0.
\end{abstract}

\section{INTRODUCTION}

\subsection{INTRODUCTION TO PROBLEM}

In the foreground interest in the field of water management still more are getting and applying options of numeric modeling, mainly based on very rapid development of the performance of personal computers. Using the numerical simulations of fluid flow, we are today able to obtain detailed information about all characteristics of the flow required for optimal design of waterwork. However, a significant disadvantage of numerical simulation is the necessity to obtain initial and marginal (boundary) conditions that must be entered as input data into the computer calculation program. These input data is in most cases can be obtained only on the basis of physical modeling, so measurements characteristics of flow on the physical model. The results of numerical simulations must be compared with the results of physical modelling and on the base it calibrated the numerical model. To determine the characteristics of liquid flow respectively velocity field on the physical model appears to be an ideal method of PIV (Particle Image Velocimetry), which allows us a sufficiently accurate measurement velocity field of fluid flow in the selected area. $[1]$.

\footnotetext{
- Ing. MISTROVÁ Ivana, DiS., Institute of Water Structures, Faculty of Civil Engineering, Brno University of Technology, 60200 Brno, Veveři 331/95, 541147 191, mistrova.i@fce.vutbr.cz, mistrova.ivana@centrum.cz

Ing. ZUBÍK Pavel, Ph.D., Institute of Water Structures, Faculty of Civil Engineering, Brno University of Technology, 60200 Brno, Veveří 331/95, 541147 296, zubik.p@fce.vutbr.cz
} 


\subsection{The method PIV (Particle Image Velocimetry)}

PIV is a measurement technique that allows to obtaining information about the instantaneous distribution of velocity in two-dimensional field in the flow fluid. The velocities are determined from the measured distance travelled by drifting particles in the flow, and the time that the particles need to overcome this distance. The particles are either part of the flowing medium (less often), or they saturate the flowing medium (in most cases). The system displays and analyzes the particles that are selected in the flowing medium light plane cut. Properly placed lighting plane is created by the system of power laser and optical elements. To create a stroboscopic effect, which allows us to "freeze" motion of particles, is the excitation of light plane pulse. The time between pulses is the time used to calculate the velocity.

A recording system, most CCD camera, which is located an optical axis perpendicular to the light plane, each particle appears as bright grains on a dark background. There are two images taken of particles drifting very rapid succession. Two images captured consecutively in the time interval $\Delta t$ are marked as double photo or double image and in the further processing to create a basic data set for evaluation of velocity.

The images are divided into rectangular areas called the examined area. For each of these examined areas image of particles captured the first image and the image of particles captured by the second film helps to create the replacement vector. The replacement vector is calculated using the cross-correlation analysis [2].

\section{MODEL OF TITLING GATE}

Measurement of velocity field round about the titling gate using the method PIV conducted in the Laboratory of Water Management Research at the Institute of Water Structures in the Faculty of Civil Engineering at the Technical University in Brno in building "F". Model of titling gate was placed in a hydraulic inclinable channel width 412 $\mathrm{mm}$. Laboratory has a separate hydraulic circuit supplied from a tank volume of $80 \mathrm{~m} 3$. Model of titling gate was placed into the first third of the length of the channel. For this model was not used the whole width of the channel, but only half of it, hence the model of titling gate had eventually length $200 \mathrm{~mm}$ (Fig. 1). This narrowing was done from the necessity to maintain the boundary conditions for the Reynolds criterion, where the value must not fall below this limit:

where:

$$
\operatorname{Re}_{\text {crit }}=q / v=5000 \text {, }
$$

$\mathrm{Re}_{\text {crit }}$ - critical value of Reynolds's criteria [-]

$\checkmark \quad$ - kinematic viscosity of water $\left[\mathrm{m}^{2} \cdot \mathrm{s}^{-1}\right]$, dependent on the temperature of the water

q - specific flow rate $\left[\mathrm{m}^{2} \cdot \mathrm{s}^{-1}\right][3]$.

Velocity field round about titling gate was measured in three positions of the setting titling gate, i.e. for 3 angles of recline $a=0^{\circ}, 60^{\circ}$ and $85^{\circ}$. For each position of the titling gate was measured velocity field for four levels of the downstream-water level $h_{d}: 0 . \mathrm{H}$ - without $h_{d}, 0.5 . H, 1.0 . \mathrm{H}$ and $1.5 . \mathrm{H}$, where $\mathrm{H}$ is the height of titling gate at the angle of recline $\mathrm{a}=85^{\circ}$. On this model has been the height of titling gate $\mathrm{H}=122 \mathrm{~mm}$. The model was located on a solid base, which allowed an increase of the downstreamwater level. The radius of upstream face of titling gate $R=2.25 . \mathrm{H}$ was chosen to match 
the standardization directive [5], which is most often propose these types of weirs. The geometry of the model is schematically illustrated in Figure 2 [1].

\section{MeAsurement OF VELOCITY FIELD ROUND ABOUt TITLING GATE}

Before the start of the measurement of velocity field using method PIV were carefully considered ways to illuminating the surrounding of titling gate and the possibilities of scanning illuminated fields by camera, so that the results of the measurements are representative as possible of the currents of flow in the selected area.

The best way to illuminate the space by laser would be through transparent bottom of the channel or the calm water level and scanning illuminated plane by camera placed next to one side of the channel. The illuminating through the water level is generally not appropriate because the ripple of water level causes deformation or complete disintegration of the illuminated plane. A variant of the lighting of the laser through the bottom taken into account, since it has a solid non-transparent bottom and only its side walls are made of glass panes attached in a steel frame and properly sealed. Another problem was that the titling gate was installed at the increased threshold, which was also made of non-transparent material (waterproof plywood). Performing a transparent hole in the bottom of the channel and transparent construction of threshold under the titling gate would be greatly time-consuming, especially financially and technically demanding, therefore from this variant totally abandoned.

After exclusion of illumination through the water level or through the bottom of the channel into account the illumination came from the right or left (out of camera view). That meant in this case inserting source of illuminating plane below the water level of flowing water. Interesting space could be illuminated in two ways, namely the location of the laser into the space inside of channel: 1 . before titling gate, i.e. in to space of aboveweir and 2. behind titling gate, i.e. in to space of under-weir. For this way was necessary to devise and have made waterproof aquarium (Fig. 3) of transparent material, which could be transmitting optic of laser is inserted into the space inside of channel so flooded with water, avoiding contact between the transmitting optics of laser with water.

From the layout of this variant, consisting of two ways of location of the transmitting optics of laser, however, revealed several problems. When the location of the laser before the mode of titling gate (Fig. 4) could be measured the velocity field only in the space behind and above titling gate because construction of titling gate is also made of non-transparent material (stainless steel sheet). Velocity field behind titling gate could be measured only the second of the above ways, the location of the laser behind the model of titling gate, i.e. in to space of under-weir (Fig. 5). From here showed that measurements of velocity field of whole interested area round about titling gate (i.e. before and behind titling gate) would be very extensive and time-consuming. And not only the measurement itself, i.e. data collection for all chosen positions of titling gate and 4 levels of lower water level associated with the relocation of transmission optics, but also to evaluate the measured data, where it would be necessary outputs, i.e. determined velocity fields before and behind the model of titling gate connect together in one image of character of the flow in whole interested area round about titling gate. Another problem, which in itself was hiding this type of arrangement transmitting optics, was the fact that the distance between the source of light and interest area was too 
large. Water flowing through the channel contained a sufficient quantity of particles for successful measurements, but it was greatly turbid. For reasons of minimizing the influence of the measured flow was necessary to put an aquarium with transmitting optics relatively far from the titling gate and it subsequently led to the problem scattering of light plane in turbid water. This problem causes the acquisition of inferior double images, whose assessments would be very difficult if not impossible, and a many of double images would most likely be excluded from the total series of measurements. The results of measurement would have become insufficiently indicative about time-medium velocity fields, for which is just needed sufficiently long series of measurements, i.e. double images.

Another problem during the testing of variants suitable arrangement transmitting and receiving optics discovered was insufficient space on the right side of the channel for the location of the recording camera to the required distance so that it scanned the whole interested area. The location of the camera to the right side it was not possible because of the need to narrowing the channel using the non-transparent structure of waterproof plywood. This problem was solved by scanning the interested area through a reflection mirror that was located at an angle of $45^{\circ}$ to the vertical wall of the channel (Fig. 6). Based on this solution could be a camera positioned longitudinally the channel and the image in the mirror was also scanned with an angle of $45^{\circ}$.

Difficulties in measurement also caused the lighting conditions of the room of the laboratory. The room is very large and fairly well lit by large windows. Therefore, in order to use the method PIV measurements of sufficient quality it was necessary to darken the room as much as possible and prevent the approach of daylight into the room (Fig. 7). To increase the measurement quality measurements were also carried out in the evening, so the influence of daylight completely eliminated.

After solving the problems listed above have been carried out the first measurement, which showed, however, that illuminating with the source below water level is almost unusable in the given circumstances. Subsequently it proceeded to attempts with illuminating of interested area through the free water level. Thanks to a very calm water level in the space above and in front of titling gate this way of lighting has proved satisfactory in the given circumstances providing the best results. For this variant of the arrangement of the optics had to be necessary fitted over the model of titling gate next mirror at an angle of $45^{\circ}$ from the horizontal plane. On this mirror attend to refraction of light plane to right-angle and transmitting optics of laser could be placed longitudinally the channel to the top of the structure of the channel (Fig. 8). The mirror had to be fitted because of the impossibility of the suspension of transmitting optics to a sufficient distance over the channel with the model so that it illuminates the whole interested area directly.

Flow field in the space behind the titling gate, due to the undulating water level, however, could not be measured by illuminating through the water level. For completeness, so it was still measured velocity field behind the titling gate, when it was used the second variant of arrangement of measuring device, and so location transmitting laser below the water level using a waterproof aquarium. The hydraulic conditions behind the titling gate allow us, without affecting the flow in the area of interest, place a watertight box closer to the measured object, and therefore significantly reduce the problem of scattering of lighting energy. In this way the area behind the 
titling gate could be measured, where during the each state in the lower water levels to occurring wavy hydraulic jump at the water level (Fig. 9) and reverse cylinder (roller) at the bottom of the channel in the area behind the titling gate (Fig. 10).

During the whole measurement, it was necessary to pay attention to the dangerous reflections of the laser light energy. When water flow over the titling gate in some modes can give rise to an aerated stream. Air bubbles then cause considerable distortion and breaking of a light plane and randomly appear strong reflections of laser light that may cause damage of the recording camera. In this matter it is always necessary to consider the ratios between the quantity and size of air bubbles, set laser power and camera distance from the object.

Practical experience with measurement in terms of flow separation from the structure that is surrounded in a situation of air entrainment into the water stream, at the same time experiences the deployment and adjustment of mirrors and measuring techniques were used also for solution of a grant project GACR No. 103/09/0977.

\section{THE MEASURING EQUIPMENT USED FOR THE MEASUREMENTS OF VELOCITY FIELDS ROUND ABOUT TITLING GATE}

\subsection{Characteristic Of MEASURING SET FOR METHOd PIV}

The main part of this set includes a double pulse laser type Nd: YAG laser type, producer of the New Wave Research, Gemini PIV type, with adjustable repetition rate from 0 to 15 $\mathrm{Hz}$ and FlowSense 2M camera, the Danish producer Dantec with a resolution of $1600 \times 1200$ pixels. To synchronize all elements in the measuring set was used a central control unit Hub System also made by Dantec. To saturation of water may not be used for the added particles because the water in the tank has been sufficiently saturated with suitable particles, but during the moving water attend to its turbidity, which was to conduct measurements using method PIV very unfavorable. Measurement of the velocity fields were carried out in one of the longitudinal section of the channel. In this chosen longitudinal section was recorded a set from 50 to 120 instantaneous velocity field states with sampling frequency of $5 \mathrm{~Hz}$ and for each position of the recline of titling gate and each position of lower water level. PIV is not intended to measure a point velocity, but to detect spatio-temporal context. On Figure 11 it is possible to see an overall view of the measuring equipment for the measurement by method PIV deployed around the channel with model of titling gate [7].

\section{THE MEASUREMENT RESULTS}

As already mentioned, the velocity field was measured only in one longitudinal section of channel, which was placed in a vertical longitudinal plane of symmetry of the channel before the titling gate, i.e. to half of the length of titling gate. The light plane was thus parallel to the longitudinal axis of the meter channel. On vector fields, which are the output of the measuring methods PIV, can best observe the existence of a separate stream of water from the surface of titling gate and its lower structure and also the creation of and the position (location) of a reverse flow. 
In case of total recline of titling gate $\left(a=0^{\circ}\right)$ is from a vector field see (Figure 12) that, on the edge of the lower threshold of titling gate attend to a very small separation of stream, which is at such a large scanning area is almost indistinctive. Furthermore, the flowing water copies the own construction of the titling gate.

In physical modelling of the angle of recline of titling gate $a=60^{\circ}$, we see the vector field evaluated from that the separation on the edge of the lower threshold is already noticeable (Fig. 13). Furthermore, the flowing liquid again follows the construction of titling gate, only at the pivot of titling gate has water tends to flow backward. And already after the construction of titling gate we can observe very slow reverse flow caused by the backwater of the lower water level on value 1.0. $\mathrm{H}$.

Vector field of last modelled position of titling gate with angle $a=85^{\circ}$ is shown in figure 14. On the edge of the lower threshold leads to a well-observable flow separation of water and in place of pivot of titling gate creates reverse cylinder. From the reverse cylinder water again follows the construction of titling gate. Behind the construction of titling gate, we can again see already faster reverse flow.

The output of this measurement of velocity fields by method PIV were files with geometric coordinates and the values of the vertical and horizontal velocities, which also served to appropriate verification, where necessary calibration of numeric model.

\section{THE CONCLUSION}

Non-contact measurement of medium-sized components at the velocity of the stream in the defined profile of channel with installed model of titling gate to allow relatively detailed analysis of the flow field, including the entirety of the secession of flowing water from the construction of the titling gate, origination reverse cylinder in the place of pivot behind the construction of the titling gate.

This flow, i.e. that the overflow water over the titling gate has been in terms of time and required simplification designed as a $2 \mathrm{D}$ problem. However, it is certain that this is actually a three-dimensional character of flow, which is very difficult test case for numerical simulation.

After comparing the results of mathematical modeling with the results of the measurement by method PIV, a method PIV is proved to be the appropriate solution for obtaining the necessary calibration and verification of numerical model. The obtained data can be used to enter initial conditions in to the numerical model during solving complex hydrodynamic problems of a similar type. Especially when dealing with hydraulic free-surface phenomena. The disadvantage of this solution is a great demand for qualified personnel. The method requires two most experienced workers. One should have a long-term experience with the measurement and evaluation of the data from the method PIV. The second should focus on the issue of the mathematical and numerical modeling and should have knowledge of computational numerical software. This project was implemented through a specific grant No. FAST-J-10-49/278 and grant project GACR No. 103/09/0977. 


\section{REFERENCES}

[1] Picka D.: The comparison of measurement by the PIV method and the numerical simulation velocity field on flap gate weir - Juniorstav 2011, Brno, Brno University of Technology - Faculty of Civil Engineering, 2011, p. 271

[2] Zubík P.: PhD Thesis - Particle Image Velocimetry, Brno, Brno University of Technology - Faculty of Mechanical Engineering, 2001, p. 7

[3] Mistrová I.: Master's Thesis - Parametric analysis of the values of discharge coefficient $m$ of tilting gate weir., Brno, Brno University of Technology - Faculty of Civil Engineering, 2010, p. 5 - 7

[4] Žoužela M.: Construction and operation of the new laboratories of the Institute of Water Structures, http://www.fce.vutbr.cz/VST/Ivv/index.html

[5] Standardization directive - Design and calculation of constructions of the titling gates, Praha, Hydroprojekt Praha, 1980

[6] Zubík P., Šulc J., Př́hoda J., Sedlář M.: Experimental and numerical modelling of fluid flow with separation - $9^{\text {th }}$ conference on Power System Engineering, Thermodynamics \& Fluid Flow - ES 2010, Plzeň, University of West Bohemia, 2010, p. $227-234$

[7] Mistrová I., Zubík P., Šulc J.: Physical modelling of flow of fluid with flow separation on inclined ramp with a slope $20^{\circ}-25^{\text {th }}$ Symposium on anemometry, Praha, Institute of Hydrodynamics ASCR, v.v.i., 2011, p. 33 - 40

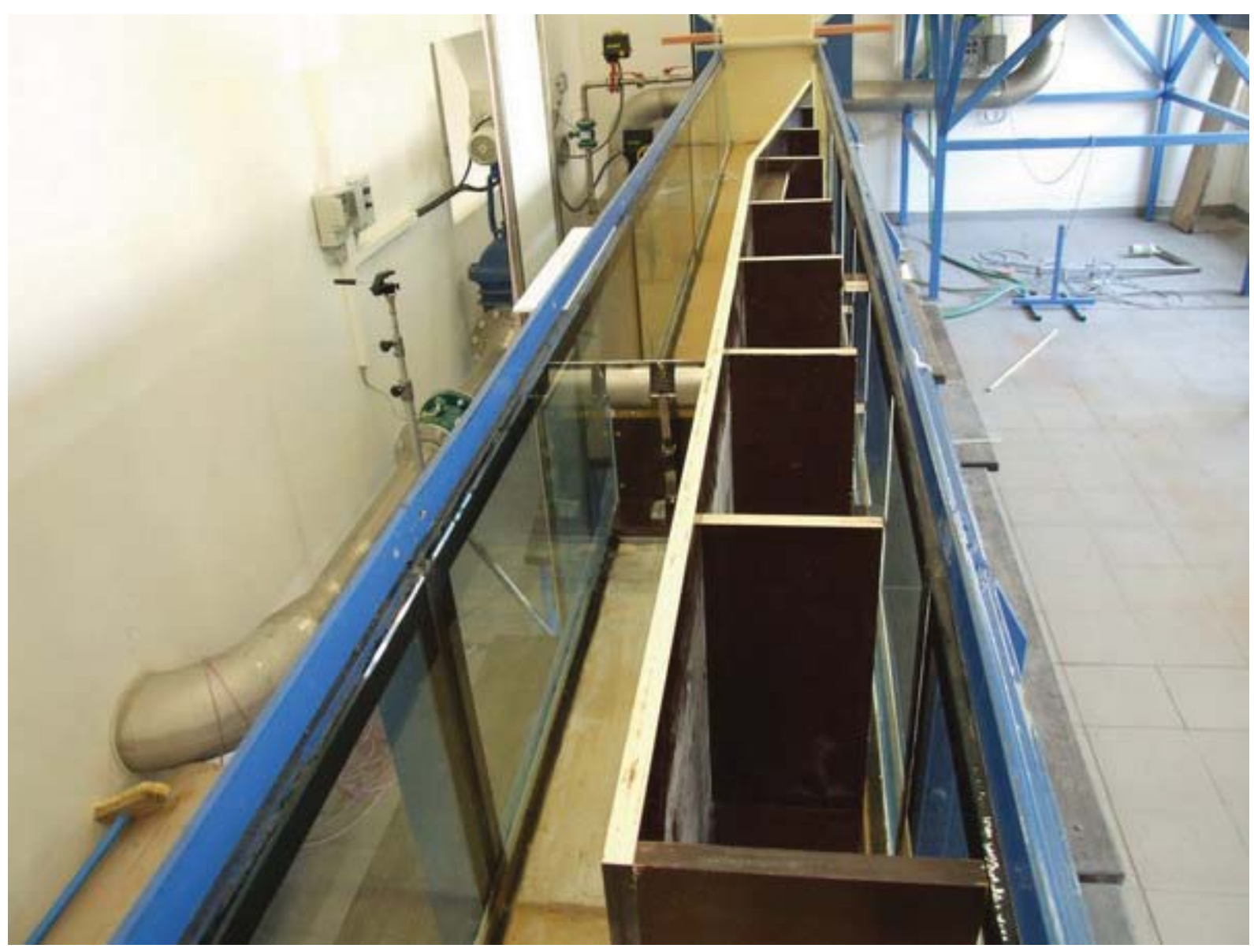

Figure 1: View of the model of titling gate in the hydraulic meter channel 


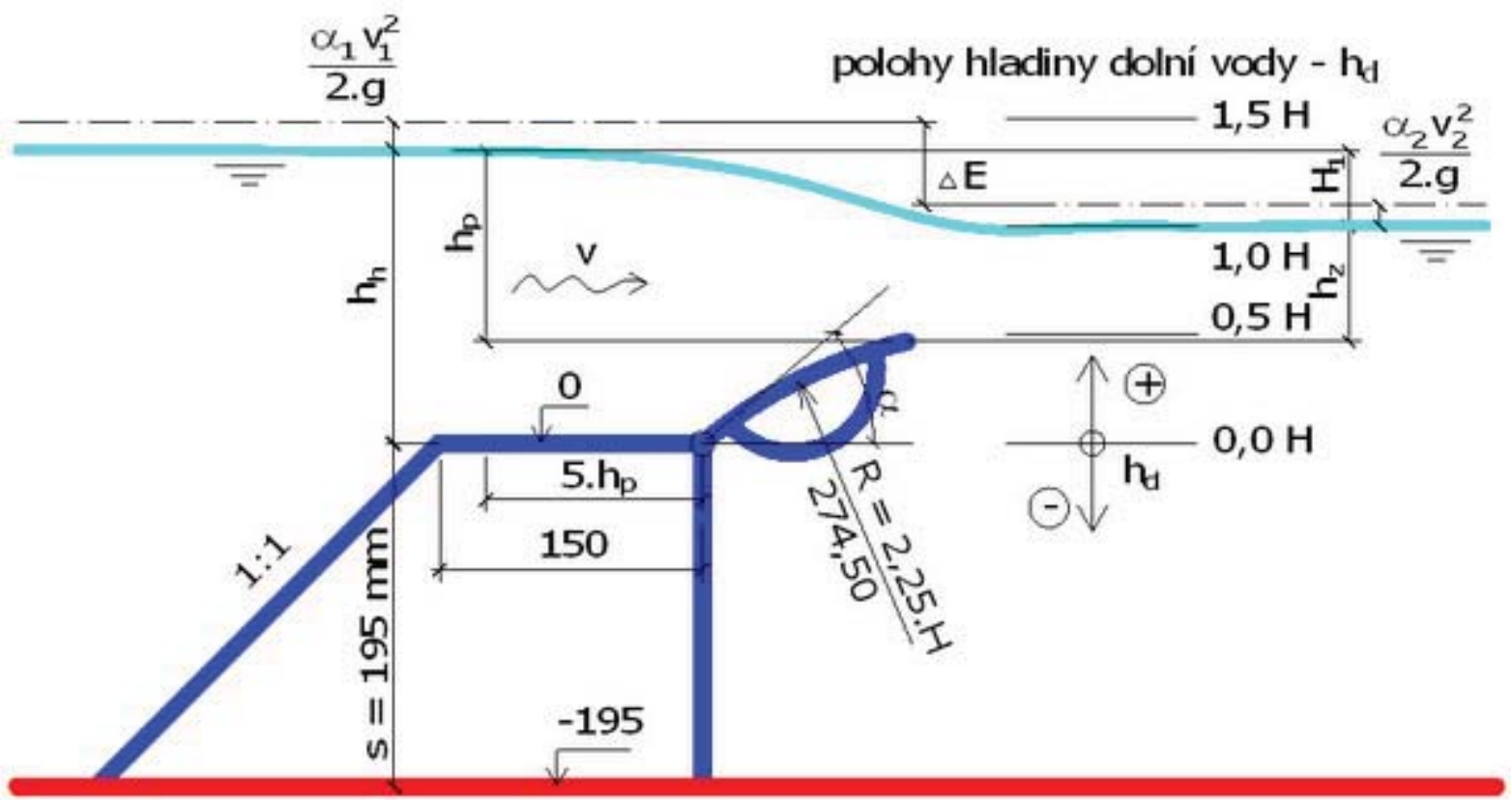

Figure. 2 Diagram of the model geometry and measurement states of the lower water levels

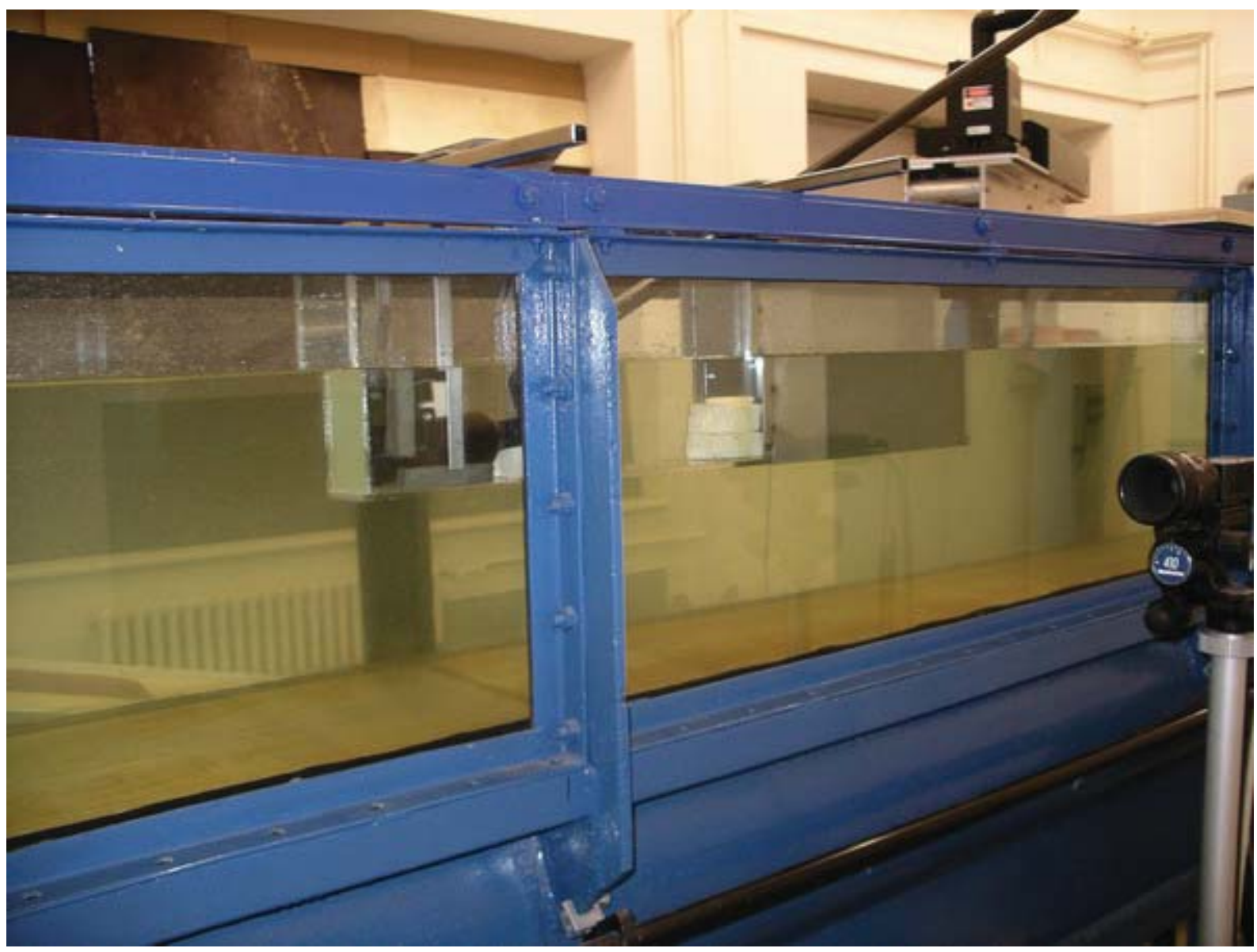

Figure 3: View of the aquarium with a transmitting optics submerged in the area of under-weir of model of titling gate 


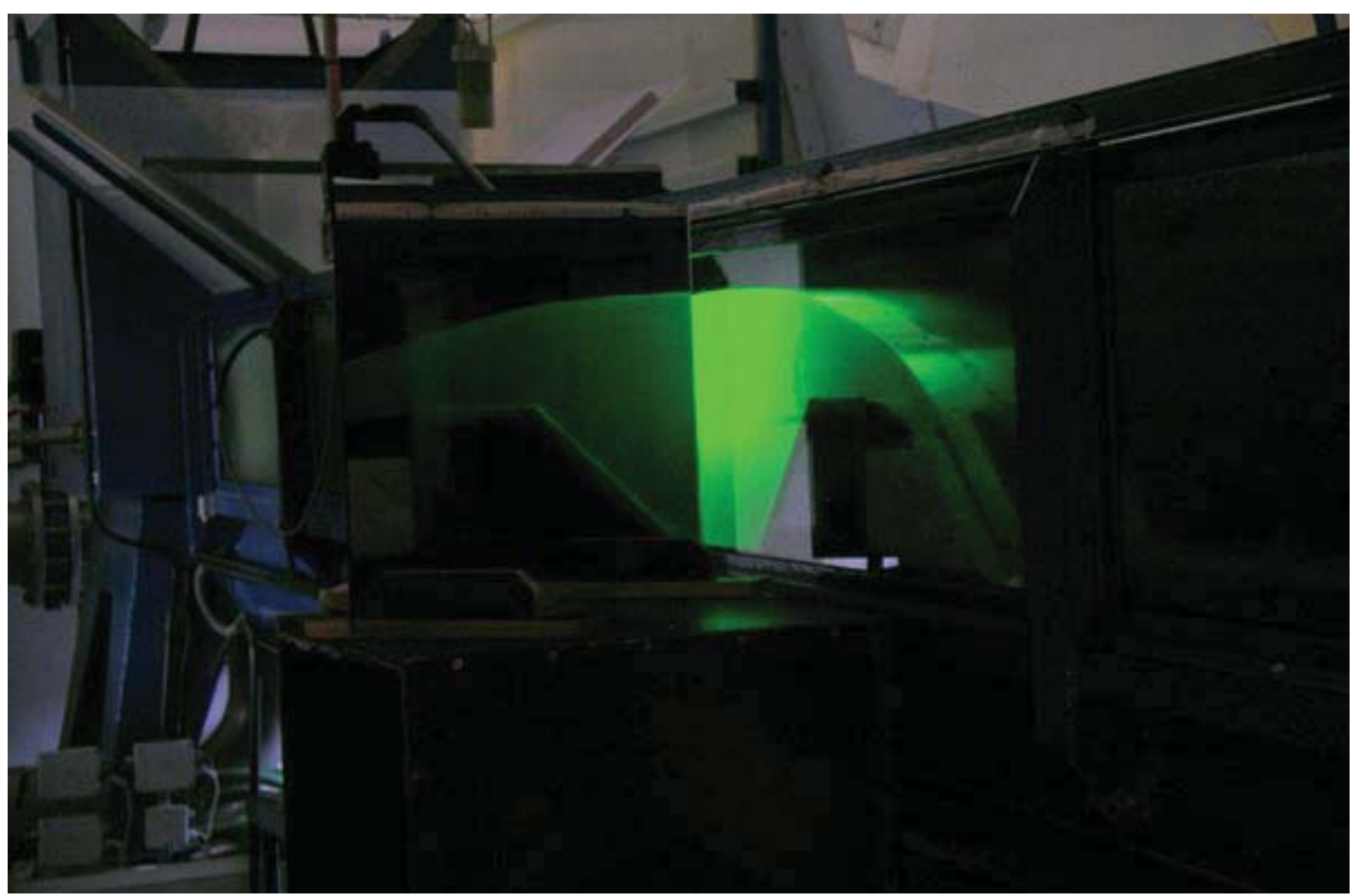

Figure 4: View of the lighted interested area when the location of the transmitting optics to before model of titling gate

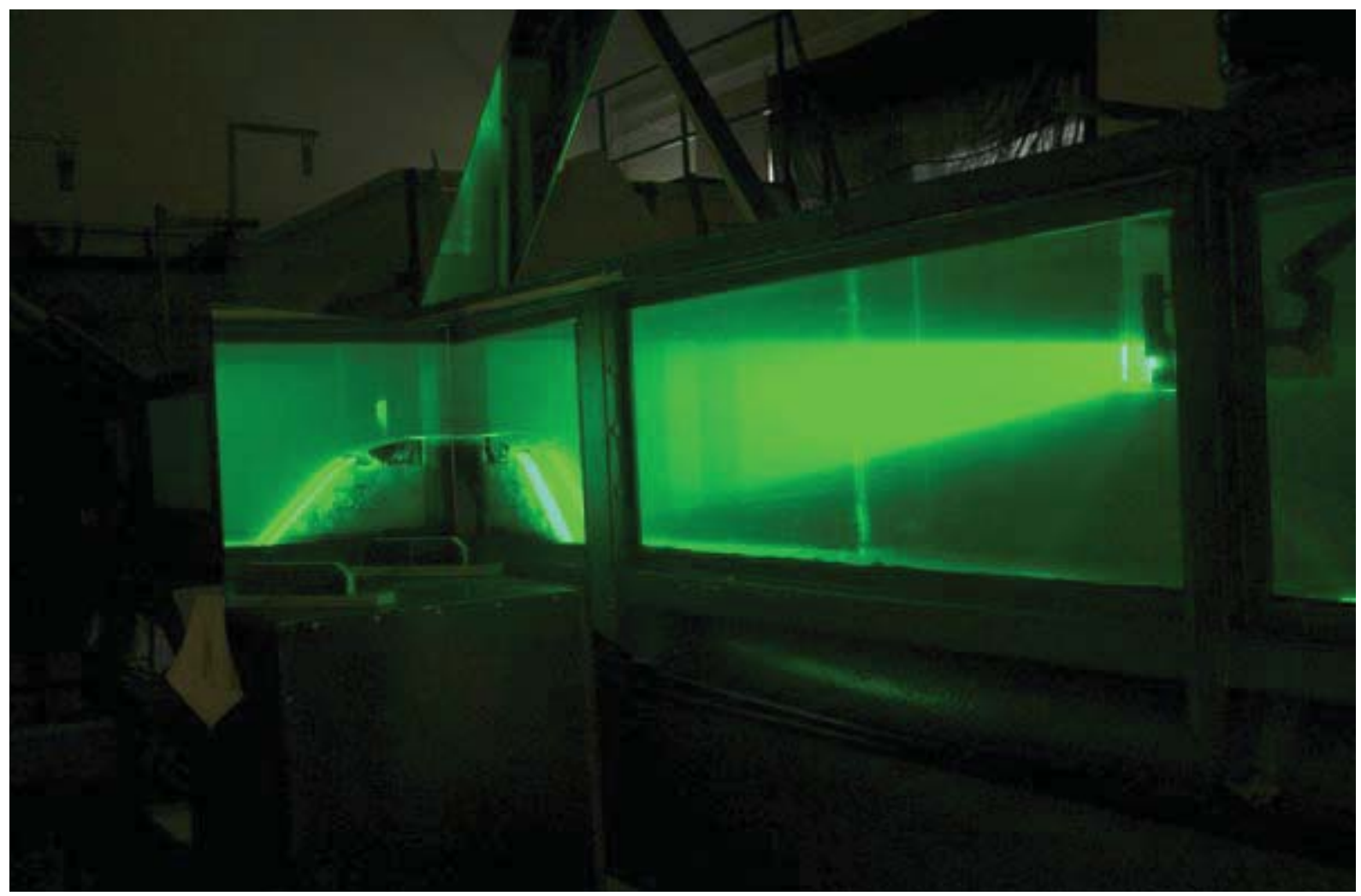

Figure 5: View of the lighted interested area when the location of the transmitting optics to behind model of titling gate 


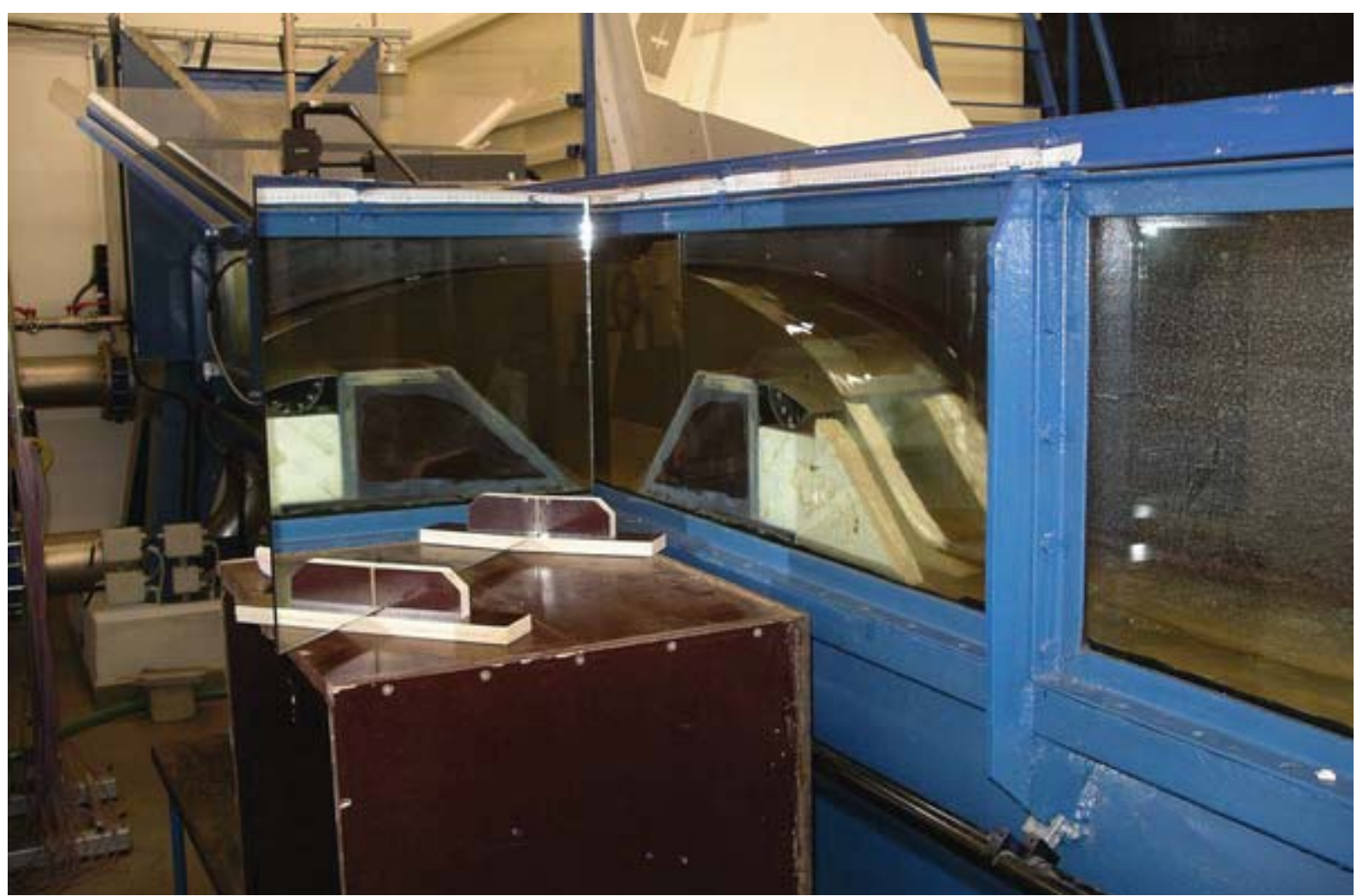

Figure 6: View of the mirror for the recording of interested area by camera

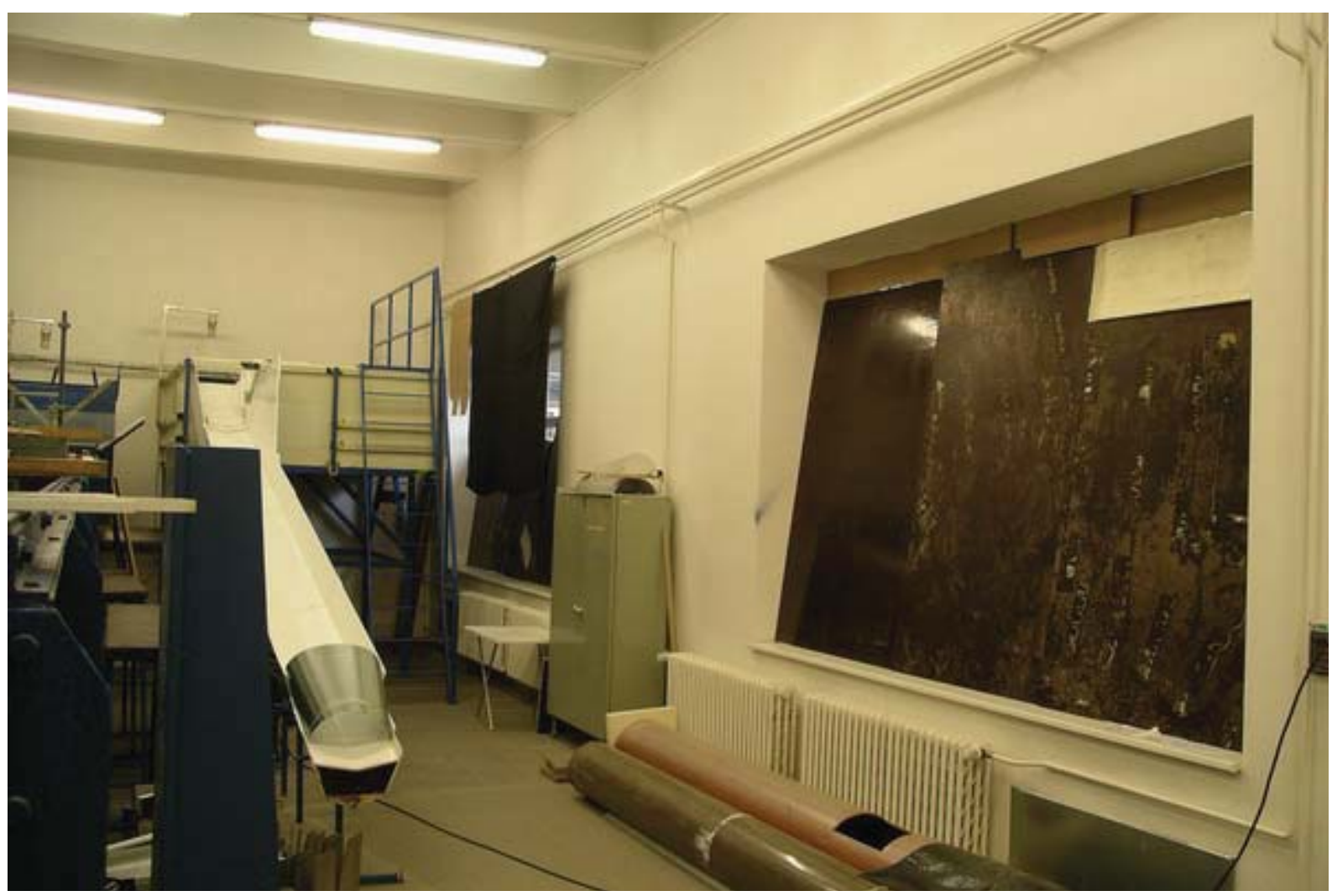

Figure 7: Darkened room laboratory for the measurement using method PIV 


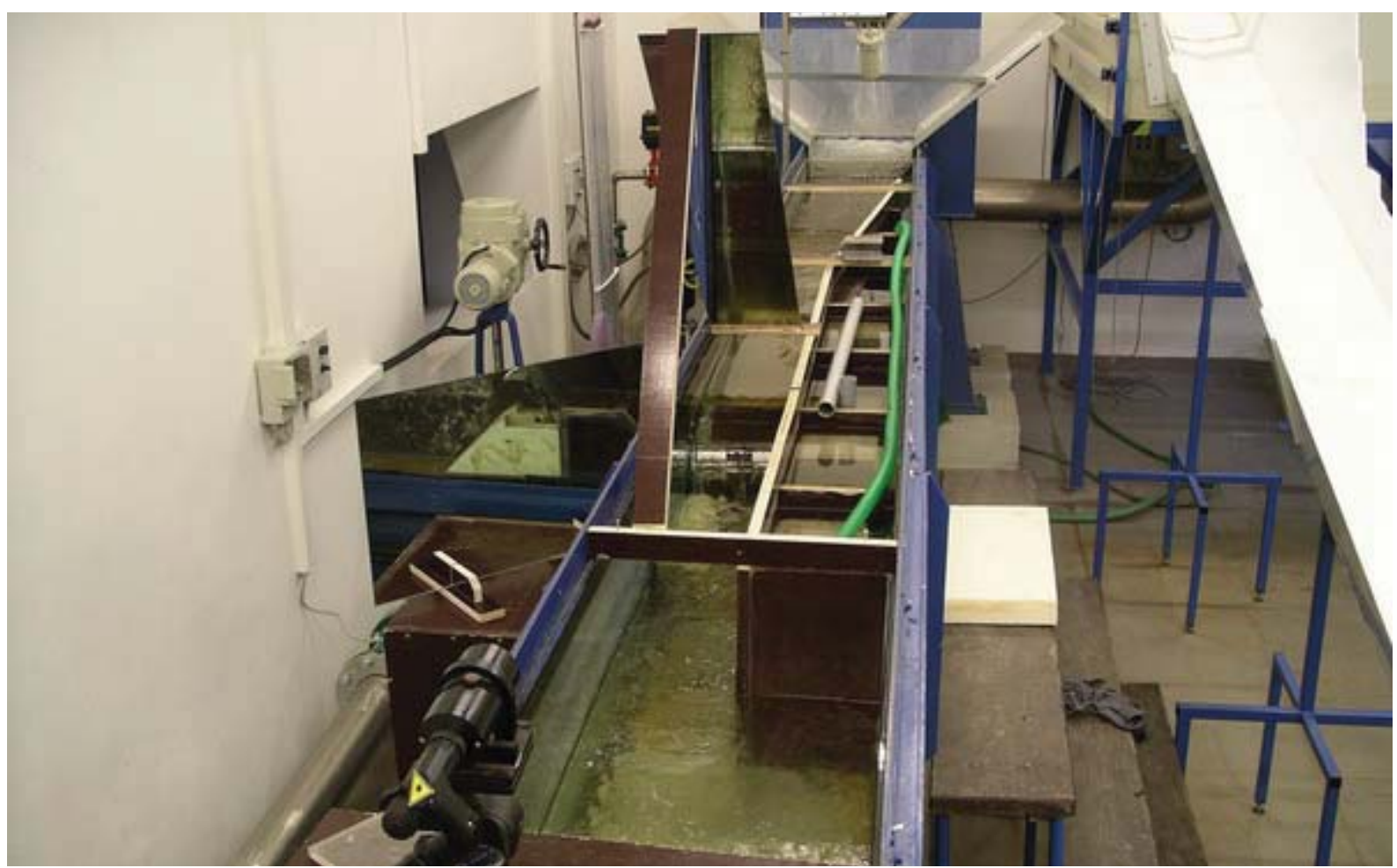

Figure 8: View of the transmitting optics of laser and mirror for break the light plane, located on the upper edges of the channel

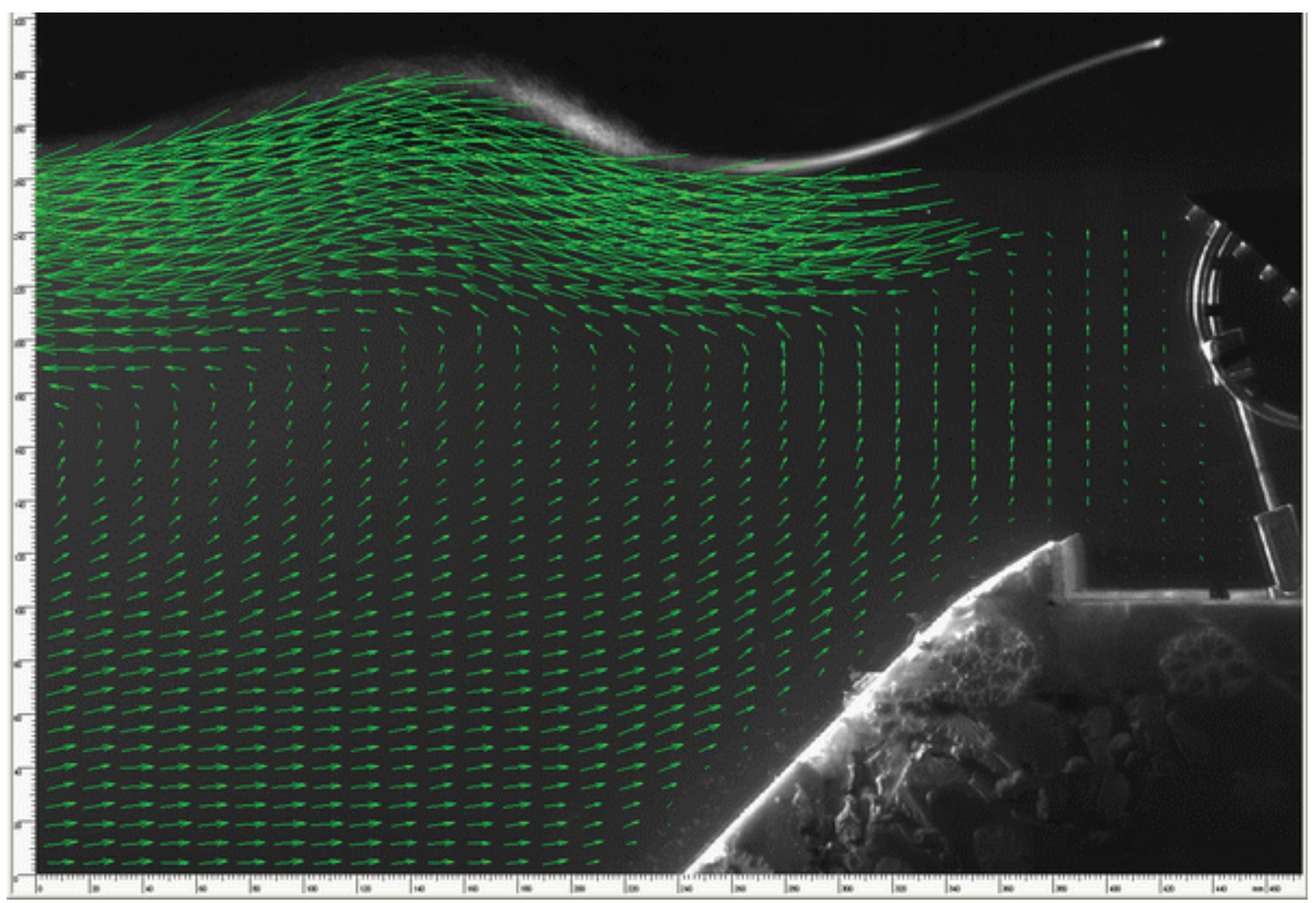

Figure 9: The image of the time-medium values of the projections of vectors of velocity to a vertical plane, in a place behind the titling gate, for the position of titling gate $a=$ $60^{\circ}$ and the position of lower water level $1.0 . \mathrm{H}$ 


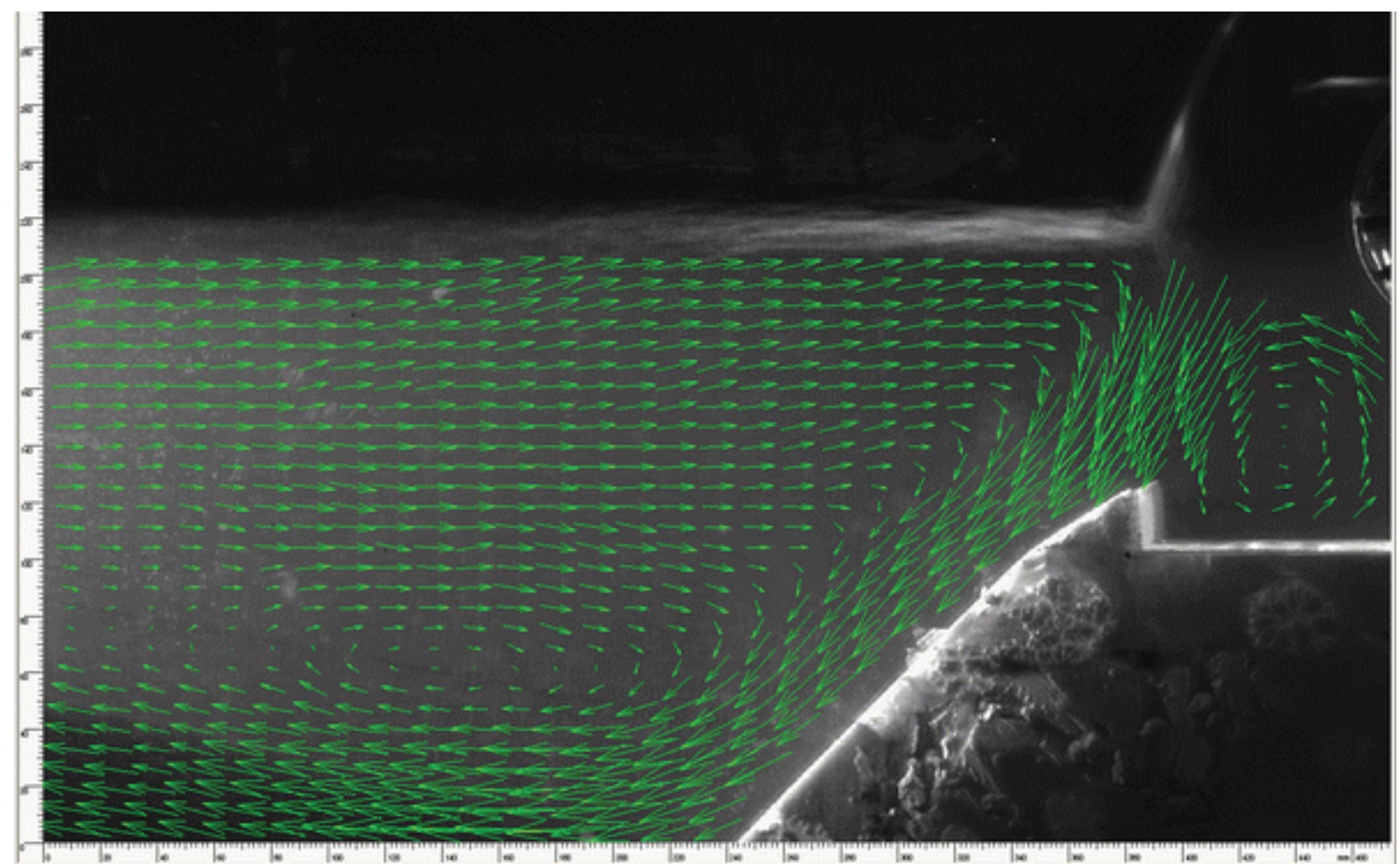

Figure 10: The image of the time-medium values of the projections of vectors of velocity to a vertical plane, in a place behind the titling gate, for the position of titling gate $a=$ $85^{\circ}$ and the position of lower water level $0.5 . \mathrm{H}$

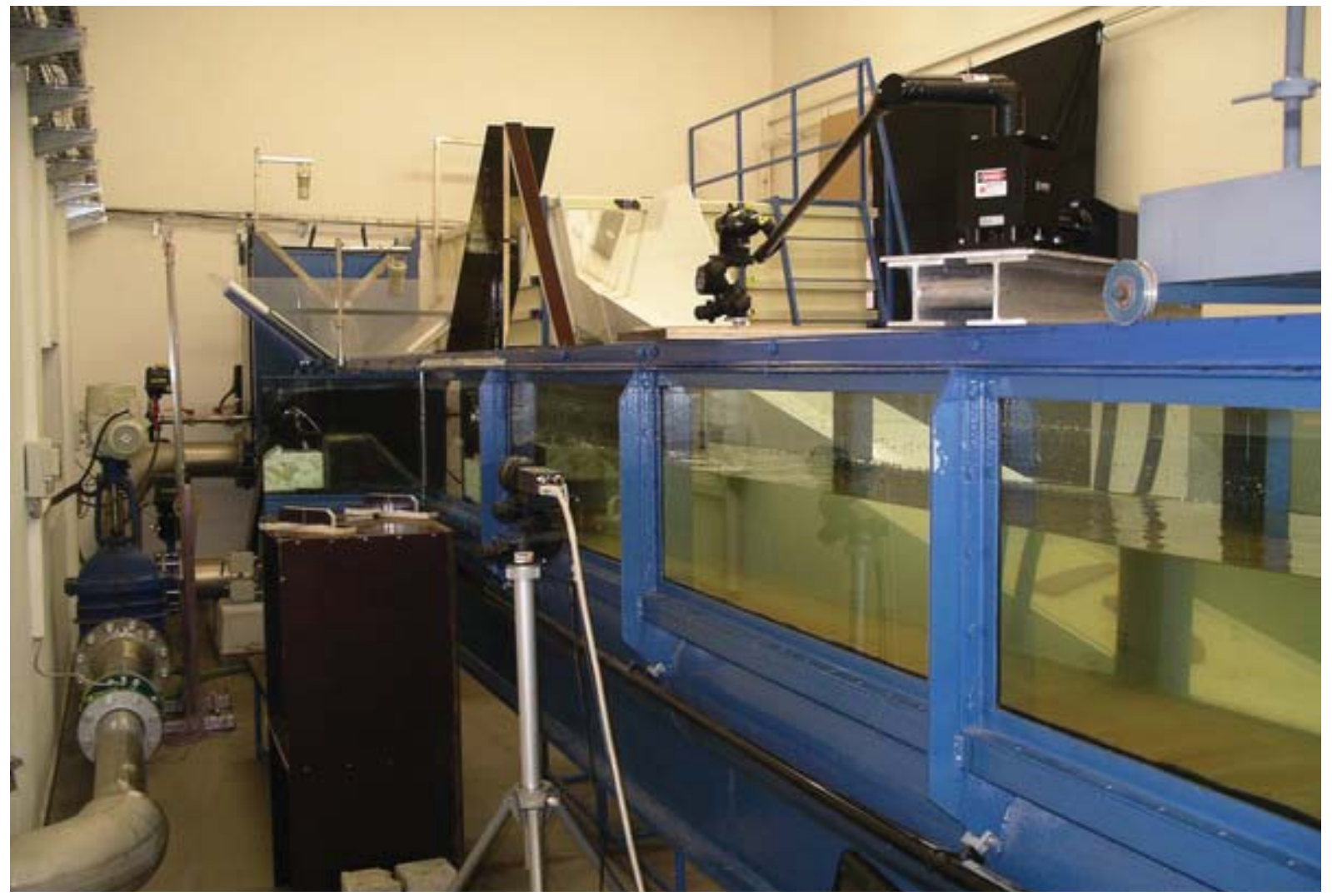

Figure 11: View of the PIV measurement technique at the water meter channel 


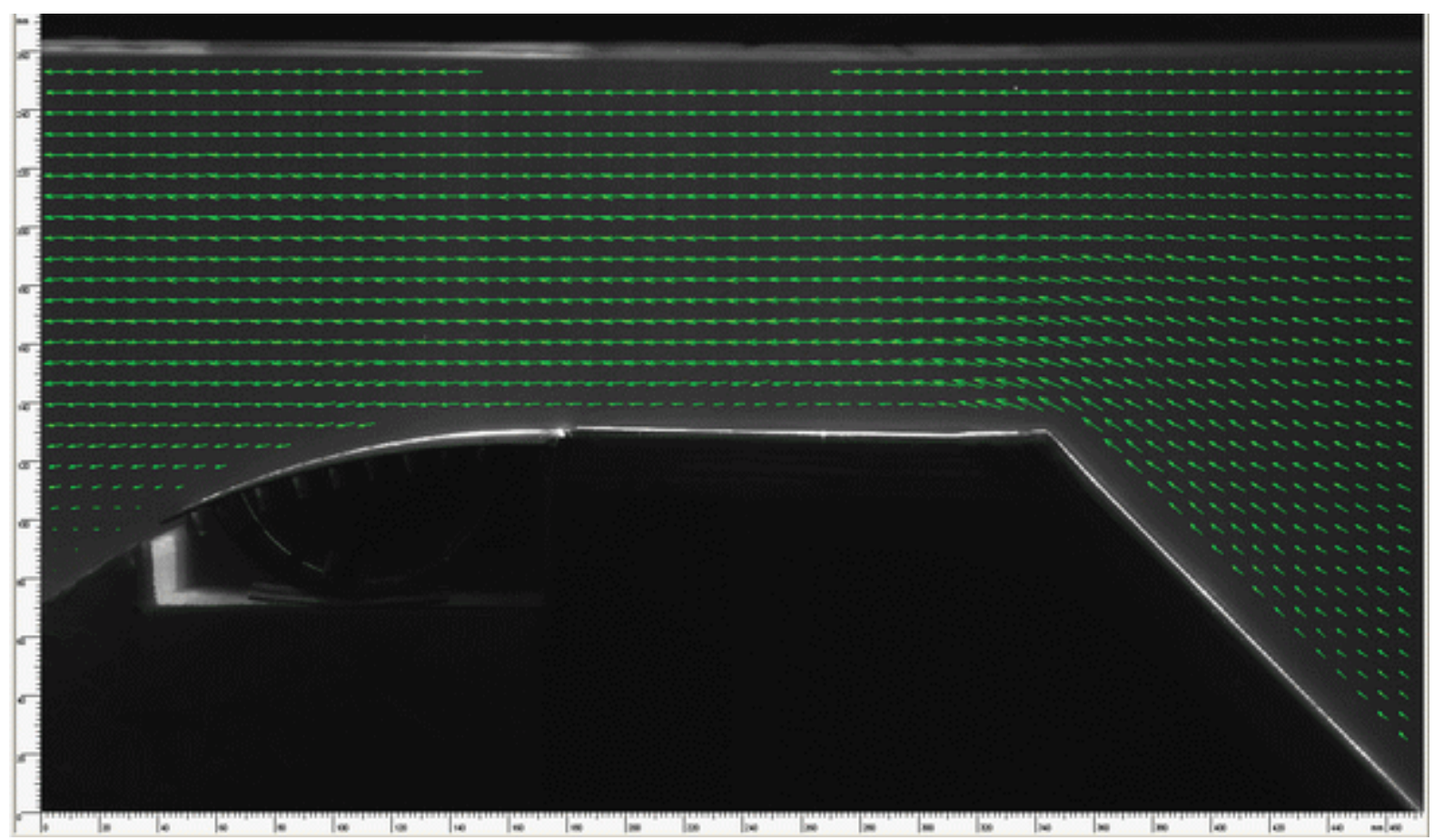

Figure 12_ The image of the time-medium values of the projections of vectors of velocity to a vertical plane, for the position of titling gate $a=0^{\circ}$ and the position of lower water level 1.0.H

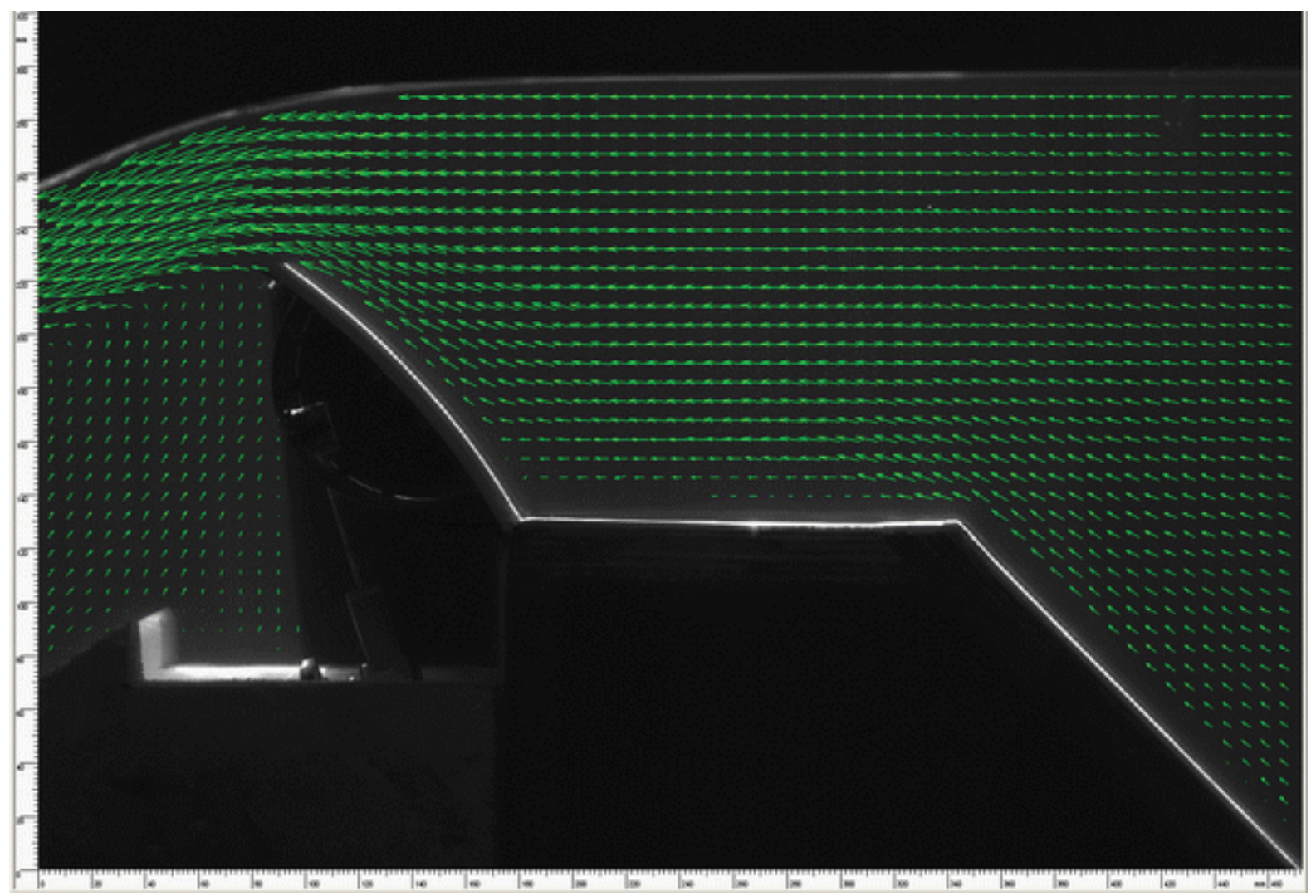

Figure 13: The image of the time-medium values of the projections of vectors of velocity to a vertical plane, for the position of titling gate $a=60^{\circ}$ and the position of lower water level 1.0.H 


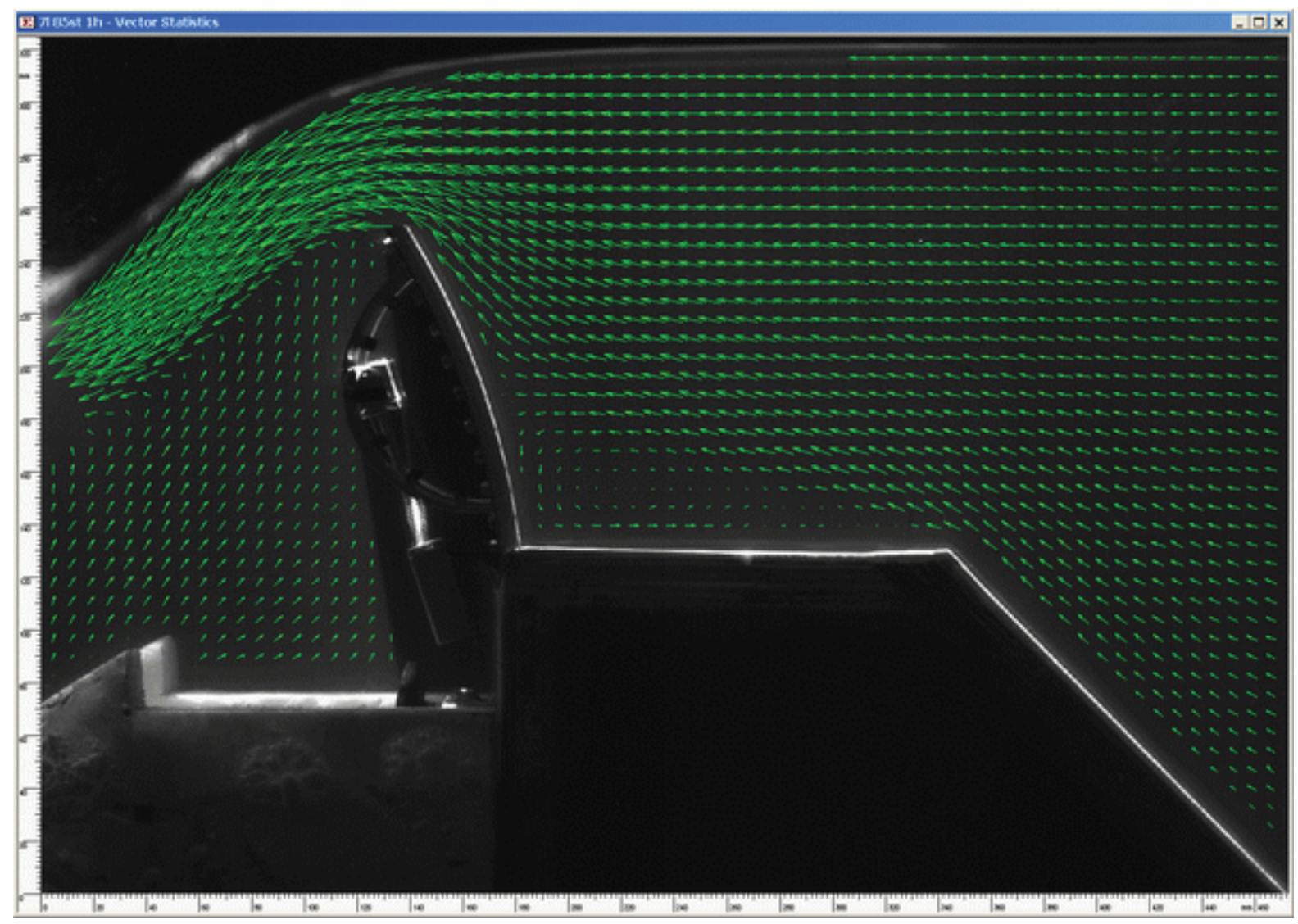

Figure 14: The image of the time-medium values of the projections of vectors of velocity to a vertical plane, for the position of titling gate $a=85^{\circ}$ and the position of lower water level 1.0.H 